\title{
Hierarchical and Flat based Hybrid Naming Scheme in Content-Centric Networks of Things
}

\author{
Sobia Arshad, Babar Shahzaad, Muhammad Awais Azam, Jonathan Loo, Syed Hassan Ahmed, Saleem Aslam
}

\begin{abstract}
Information-Centric Networking (ICN) approaches have been considered as an alternative approach to TCP/IP. Contrary to the traditional IP, the ICN treats content as a firstclass citizen of the entire network, where names are given through different naming schemes to contents and are used during the retrieval. Among ICN approaches, Content Centric Networking $(\mathrm{CCN})$ is one of the key protocols being explored for Internet of Things (IoT), names the contents using hierarchical naming. Moreover, CCN follows pull-based strategy and exhibits the communication loop problem because of its broadcasting mode. However, IoT requires both pull and push modes of communication with scalable and secured content names in terms of integrity. In this paper, we propose a hybrid naming scheme that names contents using hierarchical and flat components to support both push and pull communication and to provide both scalability and security, respectively. We consider an IoT based Smart Campus (SC) scenario and introduce two transmission modes namely (1) unicast mode and (2) broadcast mode to address loop problem associated with $\mathrm{CCN}$. Simulation results demonstrate that proposed scheme significantly improves the rate of interest transmissions, number of covered hops, name aggregation, and reliability along with addressing the loop problem.
\end{abstract}

Index Terms-Information-Centric Networking (ICN), Internet of Things (IoT), Content-Centric Networking (CCN), Content Naming, Push-Support.

\section{INTRODUCTION}

D URING 2008-2009, number of connected devices exceeded the number of humans on Earth for the first time and had introduced the concept of glorious IoT [1]. With the proliferation in the number and the usage of these low-cost and low-power connected devices in the form of mobile devices, RFID tags, sensors, actuators and smart gadgets has further assisted in the transformation of IoT from concept to reality. IoT is also considered to be the main force behind the 4th industrial revolution which helps to build smart infrastructures [2]. IoT devices are equipped with sensing, processing, actuating and transmission capabilities. When these IoT devices connect to the internet along with human internet users, production of huge amount of useful data is an obvious outcome [3]. Management of these billions

Sobia Arshad, Babar Shahzaad and Muhammad Awais Azam are with the Department of Computer Engineering (CPED), University of Engineering \& Technology (UET), Taxila, Pakistan. (email(s):sobia.arshad,babar.shahzaad,awais.azam@uettaxila.edu.pk),

Jonathan Loo is with the University of West London, London, UK. (jonathan.loo@uwl.ac.uk), Syed Hassan Ahmed is with the University of Central Florida, USA. (sh.ahmed@ieee.org) and Saleem Aslam is with Bahria University, Islamabad Campus, Pakistan. (saleem.buic@bahria.edu.pk) "Copyright (c) 2012 IEEE. Personal use of this material is permitted. However, permission to use this material for any other purposes must be obtained from the IEEE by sending a request to pubs-permissions@ieee.org."

Manuscript received Month XX, 2017; revised Month XX, 2017. of IoT devices and huge amounts of the data they produce (through TCP/IP networking model) is facing many issues [4].

Before IoT era, the researchers were trying to address the issues of traditional TCP/IP model and proposing modifications to make it suitable for future internet. Although many intermediate solutions were proposed like Content Delivery Networks (CDN) but these efforts resulted in a known concept of ICN. In addition, internet users found more interested in content from internet (i.e., it can be any host) rather than communication with any specific host. In IoT era, ICN has appeared as a promising solution of the issues of TCP/IP model. ICN consider 'content' as main element of the internet architecture which triggered several projects including DONA, Named Data Networking (NDN), CCN, MobilityFirst, CONET, PURSUIT, NetInf and CURLING [5]. Among these, $\mathrm{CCN}$ is a prominent proof of concept [6].

Basically, CCN is a pull-based (i.e., consumer-driven) communication model where service subscriber gets data irrespective of location of content provider (publisher) only when it is subscribed for a content. $\mathrm{CCN}$ communication is based on two packets 'Interest' and 'Data' which are used to request and transfer the content, respectively. $\mathrm{CCN}$ can run on any network-enabled device because of its content-centric nature. $\mathrm{CCN}$ routers uses three data structures namely Pending Interest Table (PIT), Forwarding Information Base (FIB) and Content Store (CS). PIT keeps track of entries that are waiting to be satisfied. CS stores a copy of requested data to ensure data availbility. FIB saves interface information against any name received or generated in the Interest packet. If the Interest is not satisfied at all, there will be no entry in the FIB [7].

In $\mathrm{CCN}$, content name is assigned by the publisher of the content and this name helps to cache the content at intermediate device. Fig. 1 shows the basic content propagation and retrieval used in $\mathrm{CCN}$. There are two general CCN naming schemes (1) hierarchical and (2) flat [5].

The hierarchical naming is adopted in both famous CCN and NDN architectures. The hierarchical naming assigns content name in human-readable fashion using the hierarchical structure. It is similar to URL such as locating a web page with a given URL (e.g., /www.uettaxila.edu.pk/content/a.avi). "p" is a separator between the contiguous components of a name in the given example. The advantages of hierarchical naming include compatibility with current system and aggregation of data to minimize the routing information while improving the efficiency to search in a routing table. On the other hand, the disadvantage includes the variable length of the component name. Due to variable component name length, content name becomes long and it becomes very difficult to 


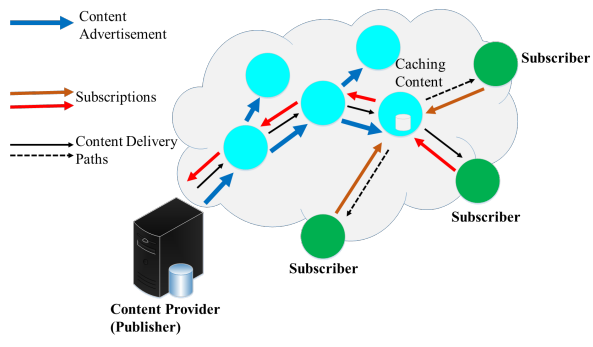

Figure 1. Basic content propagation and retrieval in CCN.

remember the long names [8]. The flat naming is utilized by the future network ICN architectures like NetInf, MobilityFirst and DONA [5]. Flat name is generated through cryptographic hashing of the content or its sub-component or its attributes. This naming procedure is unique in the sense that it assures location independence, application independence and global uniqueness. However, it slows down the aggregation mechanism (just like IP addresses aggregation) which increases the size of routing tables and their entries. Furthermore, the flat names may become unreadable which requires an additional system for the classification of readable and non-readable names.

More specifically, CCN based IoT content naming mechanism needs support for: global unique information retrieval, location independence, security, push-support and humanreadability. Push support is required when any subscriber of application wants to perform any action on content producer (i.e., sensors). Thus naming the contents by incorporating above features is one of the key expectations from $\mathrm{CCN}$ based architecture design for IoT [9] and the main motivation behind our work. Our proposed hybrid naming scheme combines both hierarchical and flat naming schemes from ICN models, add a sub-name (i.e., task) in content name to support both push and pull communication modes and appends hashed value of content to enhance security while maintaining integrity about content and its provider.

The main contributions of the paper can be summarized as follows:

- We propose a hybrid content naming scheme for CCN that incorporate both hierarchical and flat ICN naming schemes for IoT based SC.

- We introduce a sub part in the content name to support both pull and push style communication among IoT devices.

- We consider IoT based SC having heterogeneous IoT nodes (i.e., both static and mobile) to evaluate proposed naming scheme and achieve significant performance in terms of interest satisfaction rate, number of transmissions and latency, name-aggregation and security (i.e., integrity) over existing schemes [10]-[11].

The rest of the paper is organized as follows. Section II discusses the preliminaries and related work. Section III describes the proposed naming scheme while its investigation is discussed in Section IV. Performance evaluation along with results is discussed in Section $\mathrm{V}$ and we conclude the work with tentative future aspects in Section VI.

\section{Preliminaries And Related Work}

Here, we discuss few key motivations of considering CCN for IoT followed by the latest literature on naming schemes.

\section{A. CCN based IoT in a nutshell}

IoT environment have both static (e.g., sensors and machines in smart home, e-health and in smart industry) and mobile devices (i.e., connected smart vehicles). Most of IoT devices are low-power and low-cost which needs to be energyefficient, secure, reliable, interoperable and scalable. While, in $\mathrm{CCN}$, as data publisher apply naming to the content independently from its own location, this feature provides decoupling of publisher and subscriber and enables easy caching. Exploiting the $\mathrm{CCN}$ for IoT can provide aforementioned advantages over traditional TCP/IP architecture [9].

Furthermore, most of the IoT applications, contents and services require more, than only the communication between specific devices. As CCN disseminates contents and enables services through naming and this makes, naming the contents (i.e., instead of IoT devices) oftenly more significant. Next, with inherent caching and processing, IoT constraint-oriented devices can benefit even more due to their low-power nature. As in IoT environment, it is necessary to disseminate and retrieve content to multiple places with less number of transmissions, therefore caching and processing contents in the IoT local networks is important to save device battery and network bandwidth. In addition, during content request and response, caching helps to reduce delay in the result of local cache access. Moreover, caching the contents enhances the data availability where devices oftenly run out of power battery. In short, CCN approach for IoT can

1. Significantly decrease the complexity of autoconfiguration methods through naming the IoT devices, contents and services in comparison to TCP/IP (i.e., a layered protocol stack) as TCP/IP protocols including DNS, CDN, TCP, routing and IP are merged in to one $\mathrm{CCN}$ [12].

Table I

COMPARISON OF MEMORY FOOTPRINTS FOR HARDWARE AND IOT OPERATING SYSTEM

\begin{tabular}{|c|c|c|c|}
\hline \multicolumn{4}{|c|}{ IoT Operating System: RIOT } \\
\hline Module & $\begin{array}{l}\text { Developing Board } \\
\text { /Processor }\end{array}$ & $\begin{array}{l}\text { ROM } \\
(\mathrm{KB})\end{array}$ & $\begin{array}{l}\text { RAM } \\
\text { (KB) }\end{array}$ \\
\hline CoAP+RPL+6LoWPAN & \multirow{2}{*}{ Cortex-M3 } & 48.5 & 10.7 \\
\hline NDN & & 15.6 & 2.7 \\
\hline CoAP+RPL+6LoWPAN & \multirow{2}{*}{ ARM7 } & 78.6 & 8.8 \\
\hline NDN & & 22.2 & 3.5 \\
\hline RPL+6LoWPAN & \multirow{2}{*}{ MSBA2 } & 53.412 & 27.739 \\
\hline CCN-Lite & & 16.628 & 5.112 \\
\hline \multicolumn{4}{|c|}{ IoT Operating System: Contiki } \\
\hline Module & $\begin{array}{l}\text { Developing Board } \\
\text { /Processor }\end{array}$ & $\begin{array}{l}\text { ROM } \\
(\mathrm{KB})\end{array}$ & $\begin{array}{l}\text { RAM } \\
\text { (KB) }\end{array}$ \\
\hline CoAP+RPL+6LoWPAN & \multirow{2}{*}{ ARM7 } & 61.3 & 16.5 \\
\hline $\mathrm{CCN}$ & & 13 & 5.7 \\
\hline RPL+6LoWPAN & \multirow{2}{*}{ RedBee-Econotag } & 52.131 & 21.057 \\
\hline $\mathrm{CCNx}$ & & 13.005 & 5.769 \\
\hline
\end{tabular}


Table II

SUMMARY OF RELATED WORK: HN IS FOR HIERARCHICAL NAMING AND FN IS FOR FLAT NAMING.

\begin{tabular}{|c|c|c|c|c|c|}
\hline Ref. & Objective & HN & $\begin{array}{l}\text { FN } \\
\text { (Security) }\end{array}$ & $\begin{array}{l}\text { Push } \\
\text { Support }\end{array}$ & Remarks \\
\hline$[10]$ & $\begin{array}{l}\text { ICN based naming } \\
\text { for Smart Home }\end{array}$ & $\checkmark$ & $\boldsymbol{x}$ & $\checkmark$ & $\begin{array}{l}\text { Only Static Nodes considered and } \\
\text { Not for hybrid IoT environment }\end{array}$ \\
\hline$[11]$ & $\begin{array}{l}\text { ICN based Naming } \\
\text { for VANETS }\end{array}$ & $\checkmark$ & $\checkmark$ & $\boldsymbol{x}$ & $\begin{array}{l}\text { Only Mobile Nodes considered and } \\
\text { Not for hybrid IoT environment }\end{array}$ \\
\hline$[13]$ & $\begin{array}{l}\text { ICN based Naming } \\
\text { for smart building }\end{array}$ & $\checkmark$ & $\boldsymbol{x}$ & $\boldsymbol{x}$ & $\begin{array}{l}\text { Only limited Static Nodes considered } \\
\text { and Not for hybrid IoT environment }\end{array}$ \\
\hline$[14]$ & $\begin{array}{l}\text { ICN based Naming } \\
\text { for under water }\end{array}$ & $\checkmark$ & $\boldsymbol{x}$ & $\boldsymbol{x}$ & $\begin{array}{l}\text { Only limited Static Nodes considered } \\
\text { and Not for hybrid IoT environment }\end{array}$ \\
\hline $\begin{array}{l}\text { Proposed Naming } \\
\text { Scheme }\end{array}$ & $\begin{array}{l}\text { ICN based Naming } \\
\text { for Smart Campus }\end{array}$ & $\checkmark$ & $\checkmark$ & $\checkmark$ & $\begin{array}{l}\text { Suitable for IoT environment } \\
\text { with both static and mobile nodes }\end{array}$ \\
\hline
\end{tabular}

2. Offer number of opportunities to powerfully factorize functionalities (i.e., buffering and caching for the data availability and energy efficiency [15]-[16]).

3. Achieve lesser memory footprint in comparison to 6LoWPAN/RPL/IPv6 [16]. In Table. I, it can be clearly seen that ICN projects exhibits less memory as compared to TCP/IP based solutions for IoT.

\section{B. Related Work}

Most of the IoT application scenarios follow naturally CCN model features. For example, IoT sensor content retrieval and mobile content updates use naming the contents and decoupling between subscriber and publisher. These IoT scenarios get further improvements through $\mathrm{CCN}$ in-network processing, caching and light-weight memory footprints. Actually, CCN concept and its design is more feasible for IoT wild deployments in both energy-efficient and memory-efficient manner.

The naming scheme presented in [10] employs the hierarchical naming mechanism. However, this scheme does not ensure security to critical information parts or sub parts. Furthermore, the authors consider only the static nodes which limit the applicability of their naming scheme in an environment where both static and mobile nodes are present. Syed et al. [11] introduced naming scheme for vehicular ad hoc networks. They only worked for vehicles. However, they did not consider static nodes and push communication style. Safdar et al. [14] implemented NDN hierarchical naming for underwater monitoring having static nodes without security and push support. Sugang et al. [17] compared both MobilityFirst and NDN models based on two scenarios (i.e., static and mobile). However, the static and mobile scenarios are addressed separately. Moreover, reagarding mobility support, names of content remains same in $\mathrm{CCN}$ because it supports data access through content names (rather than the IP addresses), this also helps mobile users to access the data continuously even if the published content is unavailable during the content flow. Meisel, in [18] argued that current architecture of the Internet and its protocols are not suitable for an extremely mobile environment like MANET.

In summary, all of the above presented naming schemes addressed issues with a major focus on static devices without security and push support. Summary of most relevant literature along with important comparative factors is presented in Table II. Contrary to the existing naming schemes and considering
Table III

LIST OF KEY TERMS USED

\begin{tabular}{|l|l|}
\hline Key Terms & Meaning \\
\hline CCN & Content Centric Networking \\
\hline PIT & Pending Interest Table \\
\hline FIB & Forwarding Information Base \\
\hline CS & Content Store \\
\hline CO & Content Object \\
\hline
\end{tabular}

the fact that IoT needs to be able to send data and to be controlled remotely, our proposed naming scheme incorporates the security and push communication aspects in the naming mechanism for both static and mobile nodes.

\section{Proposed Naming Scheme}

In this section, we describe the design of proposed hybrid naming scheme. We combine the positive attributes of both hierarchical and flat ICN naming schemes and present a novel hybrid name scheme which has two key parts: (1) hierarchical component (2) flat component. We also discuss about the processing of interest and data messages following with the tentative advantages of proposed naming scheme. List of key terms used in this paper are listed in Table. III.

\section{A. Hierarchical Component}

This component is generated by following Uniform Resource Identifier (URI) syntax having three subcomponents: (1) Domain Name (2) Location (3) Task. Like NDN naming scheme, forward slash " " is used as a separator between the sub-components of the hierarchical component. These all three sub-components are mixed to form hierarchical component (e.g., DomainName/Location/Task can have values of uettaxila/CP/DC/action:on as shown in Fig. 2. Table IV describes the definitions for these sub-components. The hierarchical component works as a prefix to a) locate the domain name b) information retrieval site or c) the location of task to be performed. Task component represents the type of task which has two types: (1) Action and (2) Sensing. This representation provides name aggregation to optimize the routing table. The entries with a matching prefix are aggregated to minimize the number of entries in the routing table. An identifier, assigned to each aggregated entry using the arrival face of that entry, differentiates them. When an Interest is satisfied, it sends the 


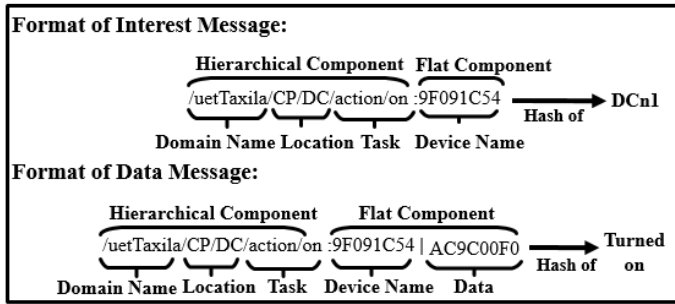

Figure 2. Example of proposed naming scheme.

Table IV

Description of The Components of Proposed NAMing SCheme

\begin{tabular}{|c|l|}
\hline Components & \multicolumn{1}{c|}{ Description } \\
\hline Domain Name & $\begin{array}{l}\text { It is used to represent the name of the } \\
\text { organization or building }\end{array}$ \\
\hline Location & $\begin{array}{l}\text { It is used to represent the location of } \\
\text { the node where to send the Interest } \\
\text { message and from where to retrieve the Data }\end{array}$ \\
\hline Task & $\begin{array}{l}\text { It is used to represent the tasks that } \\
\text { can be performed like sensing, action, etc. }\end{array}$ \\
\hline Device Name & $\begin{array}{l}\text { It is the flat part which is used to represent } \\
\text { the device uniquely by encrypting its name }\end{array}$ \\
\hline Data & $\begin{array}{l}\text { It is also flat part which encrypts the } \\
\text { data to ensure its integrity }\end{array}$ \\
\hline
\end{tabular}

retrieved Data to all the identifiers of an aggregated entry and then removes them from the routing table.

\section{B. Flat/Hash Component}

This hierarchical component is further combined with second (flat) component using ":" as separator. The flat component deals with device name and data. It has a limited length of 32-bits, which overcomes the problem of long hierarchical names. It has low aggregation but it ensures names uniqueness. To secure the device and data generated, FNV-1a hash [19], a non-cryptographic hashing algorithm is utilized. For example, considering the Computer Engineering Department of University of Engineering \& Technology (UET) Taxila, Node1 (n1) is in Data Communication (DC) lab then the pseudo-code for the algorithm is as follows:

$\mathrm{h}=$ offset-factor for each octet to be hashed

$\mathrm{h}=\mathrm{h}$ xor octet

$\mathrm{h}=\mathrm{h} *$ prime-factor

return $h$

Where ' $h$ ' represents the hash value while octet represents the octet of data. Offset-factor and prime-factor depend upon the length of hash. The values of offset-factor and prime-factor for 32-bit hash are 16,777,619 (0x01000193) and 2,166,136,261 (0x811C9DC5) respectively. The hash for DCn1 is 0x9F091C54 [20].

Moreover vertical bar "|" is used as a separator between Interest Message content name and Data Message content.

\section{CCN based Interest and Data Message Processing}

Algorithm 1 describes the reception and forwarding of Interest message in the network. When any sink (consumer) node generates interest packet to get any named-content, this sink node adds interest with identifier 0 in its own PIT and
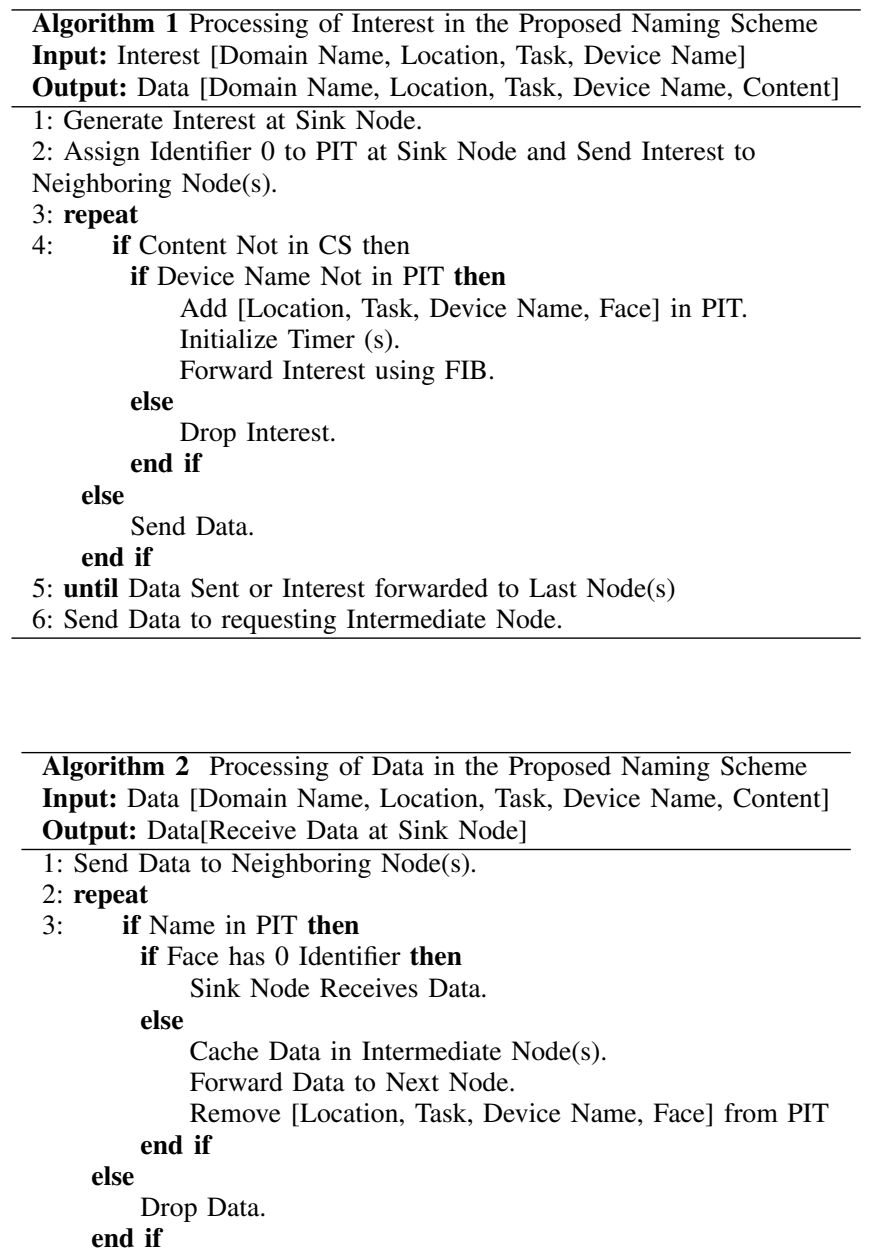

4: until Data delivered to Sink Node

5: Remove Identifier 0 from Sink Node.

forwards this Interest towards neighboring nodes. Then in the network, every node checks this specific interest in their CS and transfer data if find. If Interest is not found in CS then it checks in PIT and if found then it drops this Interest (It means that the request is already arrived for this Interest). If Interest entry is not found in PIT, then to add new entry of this Interest its location, task, device Name, and face (application name) is added along with initializing timer. And Interest is forwarded using FIB. Above process is repeated until data is sent or Interest is sent to last node in the network. Algorithm 2 describes the delivery of Data in response to received Interest. When any node finds corresponding Data to Interest, this node forwards this Data towards its neighboring nodes. And every neighboring node checks this Data Packet name exists in PIT to deliver the data. If it does not find corresponding entry, then this node drops the data (this node is not that who sent the request). If PIT entry exists then, it further checks that the packet has 0 identifier, the node has already received data. Otherwise, if this node is not consumer node then after caching the Data it forwards to next node and it removes this Interest relevant data from PIT. This whole procedure is repeated until data is delivered to sink (consumer) node. After receiving data, sink node stores and removes its identifier as 0 . 


\section{Advantages of the Proposed Naming Scheme}

We present some key advantages of the proposed naming scheme, which indicates its feasibility and viability for IoT environment and compared against related work in Table. II:

1) High Aggregation: Name-aggregation is very useful for both reducing the number of entries in the routing table and efficient lookup operation. The hierarchical part of the proposed scheme provides great aggregation; this is the one reason to use it first. For example, consider two Interests having same prefix such as /uetTaxila/CP/DC/sensing:0x 9F091C54 and /uetTaxila/CP/DC/action/on:0x9F091C54. Because the prefix /uetTaxila/CP/DC/ is same for both Interests, so they will be aggregated in the routing table, which will minimize the number of entries in the routing table and reduce memory size of the router.

2) Fixed Length: The hierarchical names, which are composed of different parts can have variable length. In return, this affects the performance in terms of query speed in routing table or forwarding table. Therefore, the names should be of fixed length. The proposed scheme has been restricted to a maximum of 102 bytes for both Interest packet and Data packet. Out of these 102 bytes, last 4 bytes (32-bits) of Interest message and last 8 bytes (64-bits) of Data are reserved for flat part. Algorithms, which depend upon the length of query, can run efficiently if the length is fixed.

3) Scalability: The proposed naming scheme considers the concept of hierarchical naming which inherits high scalability (due to high name aggregation) as compared to the current IP based communication systems.

4) Security: Proposed scheme tends to provide high level of security in form of integrity due to the use of flat component which is hashed (of device name and data message value) by FNV-1a hash algorithm.

5) Push Support: In IoT, when main application server needs to push any command towards data providers (i.e.,sensors) then proposed naming scheme's sub-component Task with the value of action provides push support.

In the following section, the proposed naming scheme is investigated for IoT based application scenario.

\section{Investigation of the Proposed Naming Scheme IN IOT DOMAIN}

To investigate the proposed naming scheme in IoT domain, we consider Smart Campus (SC) as a reference model for IoT scenarios. A SC can include temperature sensors to exhibit important feature of temperature sensing. Temperature sensing feature can be used to control and monitor both labs and classrooms through the implementation of different actions like switching the air conditioners, computers and different sensors on and off. A CCN based SC server (sink node) pulls (fetch) the data from sensors by sending Interest messages at regular intervals of time and then accordingly push (send) commands to perform above-mentioned actions.

There are different modes available for transmission of packets from source to the destination like unicast, multicast and broadcast.

\section{A. Smart Campus Scenario Description}

A hybrid model with both type of nodes (i.e., static and mobile) have been employed to analyze the efficiency in terms of sending and receiving of Interest and Data packets. Fig. 3 shows the simulation setup for the investigation of proposed naming scheme with different number of nodes as reference models. The devices used in scenario are sensors, routers and mobile data carriers for delivery of Interest messages to static nodes that are out of range. In reference model, there are eleven departments named Civil (CE), Mechanical (ME), Electrical (EE), Electronics (EC), Computer/Software (CP/SE), Transportation (TRE), Transport Office (TR), Library (LB) and Admin Block (AD), Telecom/Computer-Science Dept. (TE/CS), Industrial/Humanities Department (IE/HU). In each building, total five devices are placed at different locations for sensing temperature or switching on/off devices as mentioned in Interest packet. Out of these five devices, four are Zigbee based Temperature sensors and one is Wifi Access Point (AP). Eight mobile data carriers are used which will travel on specific routes to forward and collect the requests and data respectively. A sink node (wifi) as SC server is placed in the $\mathrm{CE}$ because this is located at the center of all other nodes. Two mobile nodes and all the nodes in CE are placed in the range of sink node as shown in Fig. 3. All nodes except sink node work as a source of required content. We vary number of mobile nodes from two to eight and mobile nodes are used to forward Interest message towards static nodes deployed in other departments. In the shown scenario with eight mobile nodes, the sink node generates an Interest; the two mobile nodes receive it and start moving towards the other two (in both directions) that are in idle state.

Second scenario (Right side of Fig. 3) having only static nodes is used for comparison purposes. If a node cannot satisfy the Interest, it forwards to other nodes in its range. This chain is used to forward the Interest from the sink node to last source node in the given scenario.

\section{B. Communication Loop Problem Solution}

As CCN implements broadcast mode of transmission in order to send and receive Interest and Data packets respectively. This broadcast mode of communication presents a problem called 'loop problem'. When a sink node transmits an Interest to all nodes in the network range and stores an entry in its own PIT. If the receiving nodes cannot satisfy the incoming Interest, they store that Interest in their PIT and then forwards to other nodes. In that case, whether an Interest is satisfied or not, it remains in PIT of the sink node, which creates a loop. For example, if a sink node has two neighboring nodes and it broadcasts an Interest towards both of them. If the Interest is satisfied on the first node but not on the second. Then the first node sends $\mathrm{CO}$ towards both sink node and second node, while second forwards the Interest by broadcasting again towards the first node and remaining neighboring nodes. Both first node and sink node will receive same interest again. This situation creates a communication loop [21]. To resolve this problem, we use the concept of unicast using multi-hopping along with broadcast. For example, in the reference model, initially, sink 

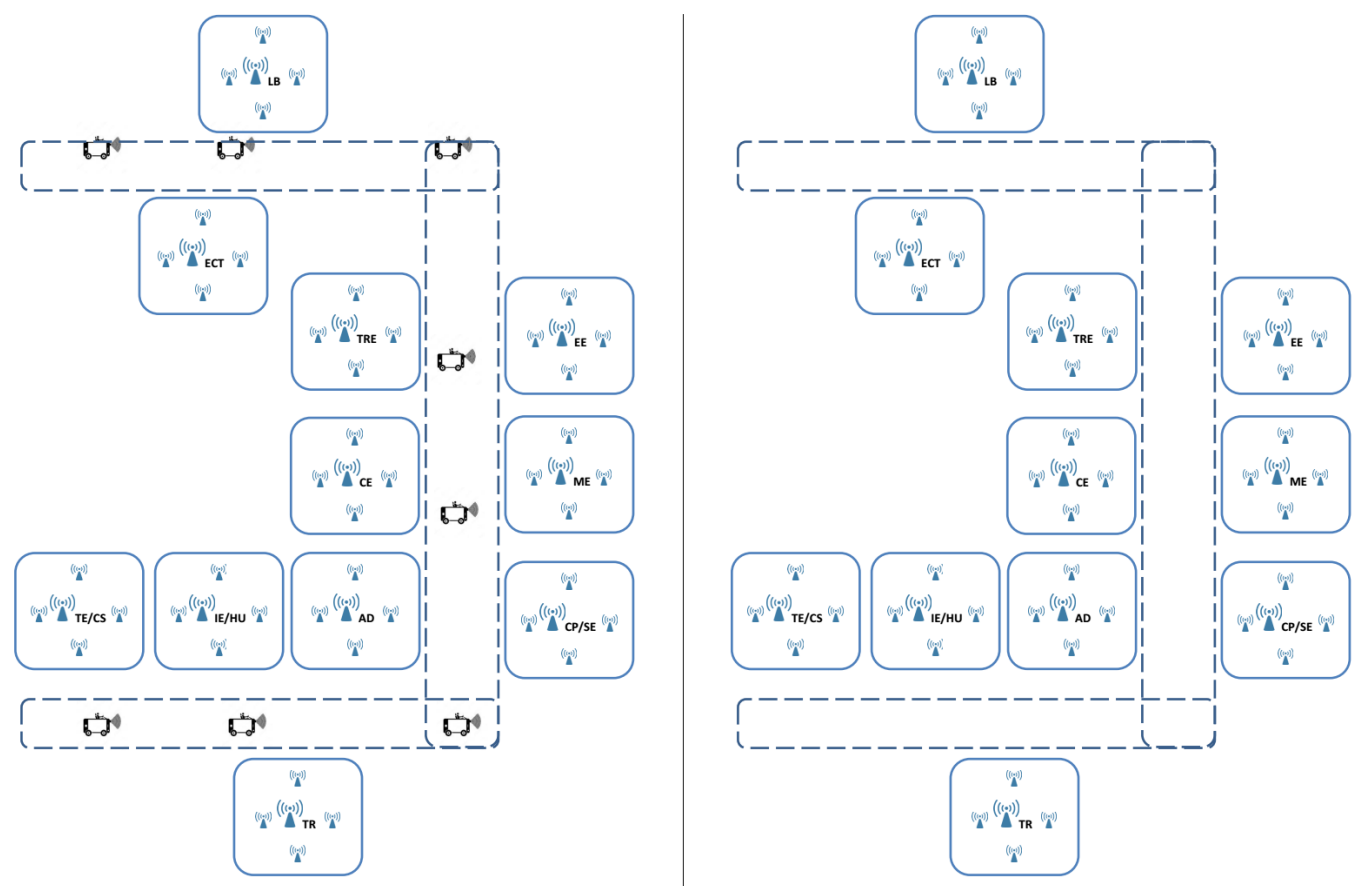

Figure 3. Reference model for proposed naming scheme: Left Side::with 8 mobile nodes and 55 Static Nodes and Right Side::only static nodes, LB=Library, ECT=Electronics, EE=Electrical Eng, TRE=Transportation Engg., ME=Mechanical Eng., CE=Civil Eng., CP/SE=Computer/Software., AD=Administration Block. TE/CS=Telecom/Computer-Science, IE/HU=Industrial/Humanities, TR=Transport Section.

node broadcasts an Interest message to all neighboring nodes, while multi-hop unicast is implemented for both to deliver the Interest packet to nodes in the department (on the basis of prefix mentioned in hierarchical component) and send Data packets back to the sink node.

\section{Tree Structure of the Proposed Naming Scheme}

We illustrate an example of proposed naming scheme (Details in Table. IV) through tree structure shown in Fig. 4. We can easily extend this by adding new locations and types of task. For example, an action can be performed on computers/air conditioners by sensing the temperatures of said location. An Interest message, e.g., /uetTaxila/CP/DC/action/on:C60B9850 is used to turn on the PC1 in DC Lab of Computer Department in UET, Taxila. After the Interest is satisfied, a Data packet will be sent back consisting of the name same as Interest and a 32-bit hashed value of a short string representing that the Interest has been satisfied or not. If the Interest is not satisfied, the payload of Data packet may have a specific hashed string to represent any malfunctioning of computer.

\section{Processing of Interest and Data Message}

Fig. 5 describes the exemplary scenario to highlight the working of the proposed scheme. For the sake of simplicity, it shows only the nodes that are involved in communication. In this example, the sink node generates an Interest message and broadcasts it to all the nodes in its range. MN1 receives the Interest message and stores it in PIT. If there is another entry with the same prefix, then it discards the Interest and adds a face identifier to the existing entry representing the original

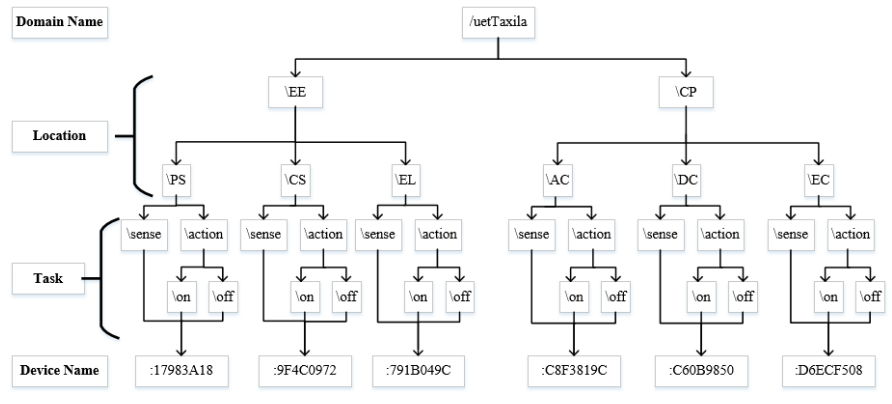

Figure 4. Tree structure for proposed naming scheme.

source of the Interest packet. Once the Interest is stored in PIT, node forwards it to other nodes in the connectivity. A mobile node is used for propagating the Interest in the network so that the nodes at a distance can receive the Interest message and perform operations accordingly. Fig. 6 shows different steps to understand the working of example scenario. Following are the steps of Interest generation and satisfaction process in the reference model:

1. As sink node is placed in CE department having one mobile node MN1 and all static nodes of CE department are in its range. Sink node generates an Interest with name /uetTaxila/EC/DC/action/on: C60B9850. It is stored in the PIT before propagation to other nodes connected to sink node. An identifier 0 is attached to the Interest stored in PIT of sink node indicating that it is the original requester of Data.

2. Sink node transmits (broadcasts) generated Interest message to all the nodes in its range (MN1 and all static 


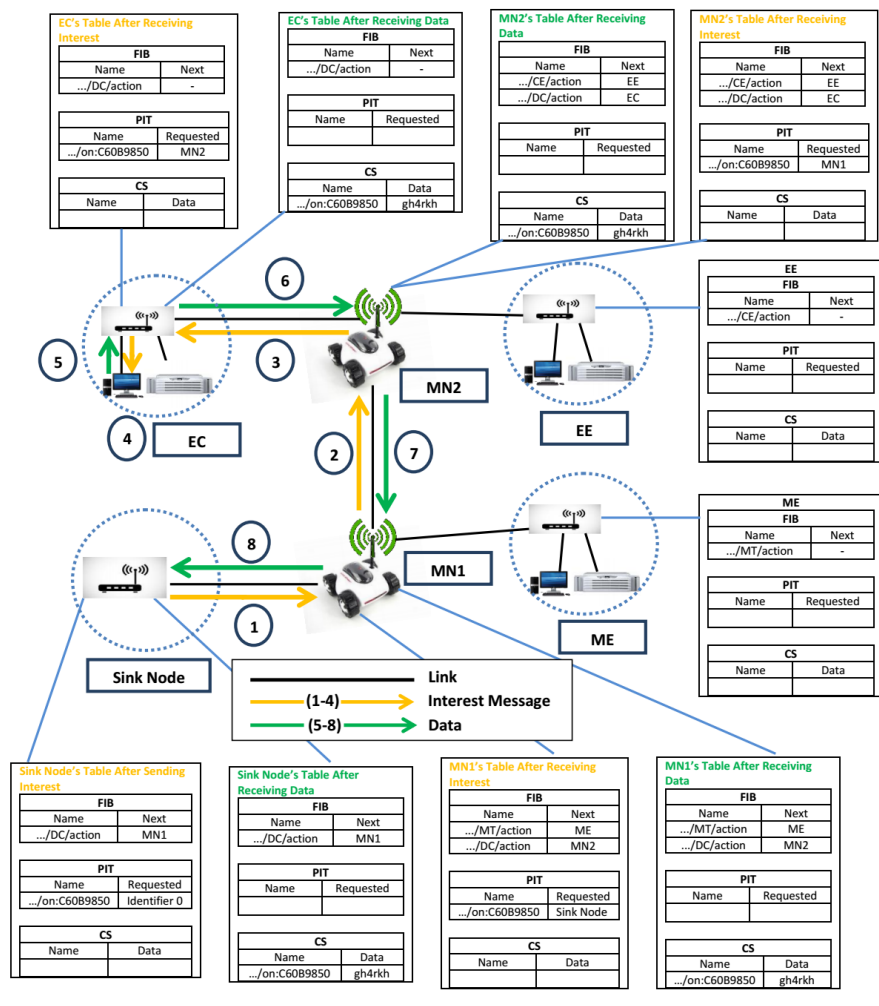

Figure 5. Working of proposed naming scheme in reference model.

nodes of CE). As in this case, static nodes which resides in $\mathrm{CE}$, discard this message by looking at the prefix. If the request is to retrieve data from nodes in other departments, mobile nodes help to forward the Interest message to nodes that are not in direct range of sink node. Therefore, MN1, after receiving Interest message from sink node, performs a long prefix match on FIB to check either this entry is already available or not. In the case of absence, MN1 starts moving on the specified route and forwards the Interest to all the faces stored in its FIB at regular intervals of time. But if the Interest is meant to perform a task in CE department where sink node resides, then Interest is processed without the interference of any mobile node. CE static node receive Interest and send corresponding Data message after performing specified action in unicast mode.

3. Nodes at EE and ME do not process the Interest because their prefix doesn't match with the Interest prefix. The mobile node, MN1 forwards the Interest message to MN2 after entering in its range and start moving towards the EC, which is the destination location in this case.

4. Five static nodes in EC department receive the incoming Interest but only one can perform the action requested in the Interest message, i.e., turning on the computers in DC Lab in Electronic Department. It is because the nodes residing within the department implements the unicast mode of transmission.

5. After the Interest is satisfied, a Data message with Content Object (CO) is sent back to requesting node. The $\mathrm{CO}$ is /uetTaxila/EC/DC/action/on:C60B9850—gh4rkh, where the prefix name of both the Interest message and CO message is same. The last hash/flat component of Data message contains the actual $\mathrm{CO}$ value.

6. PIT of EC removes the Interest from the list of unsatisfied Interests, stores the Data in its CS and then forwards to $\mathrm{MN} 2$.

7. CO message is replicated at intermediate nodes, MN2 and MN1, for fast retrieval of data in future.

8. The reference identifier 0 in PIT is removed, indicating that Interest has been satisfied. Finally, CO message is delivered to the sink node and stored in its CS indicating that action is performed successfully.

If there is a need to perform an action on a different number of nodes having same name prefix, then a prefix is sent as a name in the Interest messages instead of specifying a node. An Interest message with name /uetTaxila/EC/DC/action/on will turn on all the computers in DC Lab of EC department.

\section{Performance Evaluation}

In this section, we discuss performance evaluation of proposed naming scheme considering scenarios of smart campus shown in Fig. 3.

\section{A. Simulation Environment}

We use 55 static nodes and vary the number of mobile nodes (from two to eight) to cover the total area for exchange of Interest and Data messages among sink node and source nodes.All nodes are arranged in the form of considered scenario. CCNx implemenations are used in Contiki OS based Cooja simulator [21]. We implemented proposed naming scheme in CCNx implementations in Contiki OS based Cooja simulator installed in a Linux Ubuntu running on Virtual Machine. A core-i5 PC with 4GB RAM is used for implementation and performance evaluation. $\mathrm{CCNx}$ is modified in accordance with the proposed naming scheme. $\mathrm{CCNx}$ hierarchical naming is concatenated with flat naming by implementing a separate function to calculate hash (pseudo code is given above). Simulations are run for 1600 s and Tmote Sky type motes are used. We use Random-Waypoint Mobility Model (RWMM) mobility plugin in this simulation for mobile nodes to move in particular direction. RWMM has the following input parameters: node number, time in seconds, $\mathrm{x}$-coordinate, $\mathrm{y}$-coordinate. The node number is not the Rime address and it starts from ' 0 ' means the 'node 0 ' represents the 'mote 1'. The coordinates have value in meters. Rest of the simulation parameters that help in evaluating the proposed technique through a series of simulations in Contiki-based Cooja Simulator are summarized in Table V. Though fixed number of nodes are considered in these scenarios but this implementation can also be validated on a large scale as the proposed scheme presents high scalability.

To compare and show the worth of proposed naming scheme, three different scenarios (according to Fig. 3) are simulated in Cooja Simulator. Fig. 6 shows the simulation of only static nodes in which intermediate nodes forwards the Interest packets. The simulation of delivery of Interest message and Data message using a different number of mobile nodes is 
Table V

SIMULATION PARAMETERS

\begin{tabular}{|c|c|}
\hline Parameter & Value/Name \\
\hline Communication Stack & CCN \\
\hline Radio Medium & $\begin{array}{c}\text { Unit Disk Graph Medium } \\
\text { (UDGM): Distance Loss }\end{array}$ \\
\hline Topology Size & 200*350 \\
\hline Number of Nodes & 55 Static, 8 Mobile \\
\hline Number of Transmissions & 200 \\
\hline Tx Range & 60 meters \\
\hline Interference Range & meters \\
\hline Packet Size & IEEE 802.15.4 \\
\hline MAC Layer & ccn_driver \\
\hline Network Driver & csma_driver \\
\hline MAC Driver & sicslowmac_driver \\
\hline Radio Duty & cc2420_driver \\
\hline Cycling Driver & Tmote Sky \\
\hline Radio Driver & No limit speed \\
\hline Mote Type & 1600 s \\
\hline Speed & Random-Waypoint Mobility \\
Simulation Time & Model (RWMM) [22] \\
\hline Mobility Model
\end{tabular}

shown in Fig. 7 and Fig. 8. Each Figure contains three parts. Part 1 shows the start of simulation when the sink node sends the Interest to its neighboring nodes. If the nodes cannot satisfy the Interest, it is forwarded to other nodes using mobile nodes (part 2). Part 3 shows the case when the Interest is forwarded to the last nodes in the campus area and Interest forwarding is finished in this step.

\section{B. Performance Metrics}

The performance of proposed scheme is evaluated through following metrics:

- Satisfaction Rate (SR): the ratio of total number of satisfied Interests to total number of generated Interests and can be calculated by using equation 1 as follows:

$$
S R=\frac{\sum_{1}^{N} \text { SatisfiedInterests }}{\sum_{1}^{n} \text { GeneratedInterest }} * 100
$$

Where ' $\mathrm{n}$ ' is the total number of generated Interests and ' $\mathrm{N}$ ' is the total number of satisfied Interests.

- Average Latency: is the ratio of total latency to the total number of packets received at sink node and can be calculated using following equation 2 :

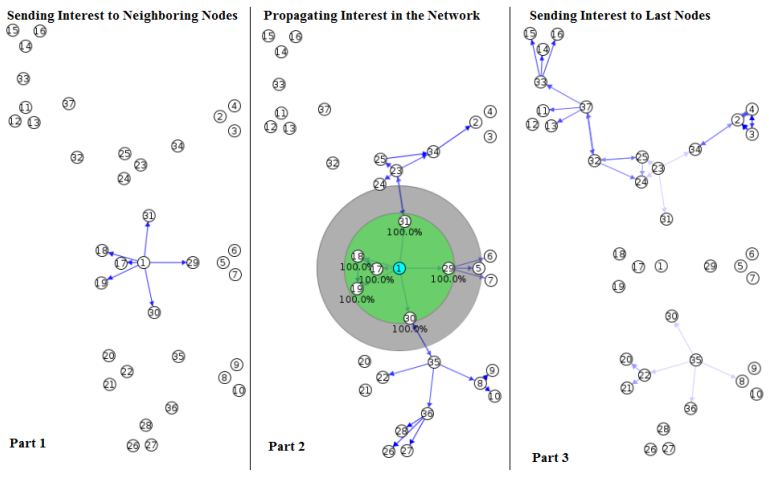

Figure 6. Simulation scenario 1 (only static nodes).

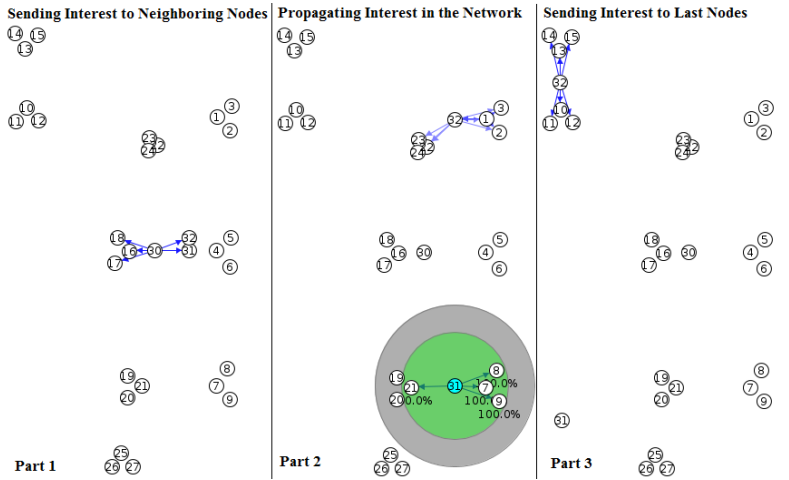

Figure 7. Simulation Scenario 2 (2 Mobile Nodes).

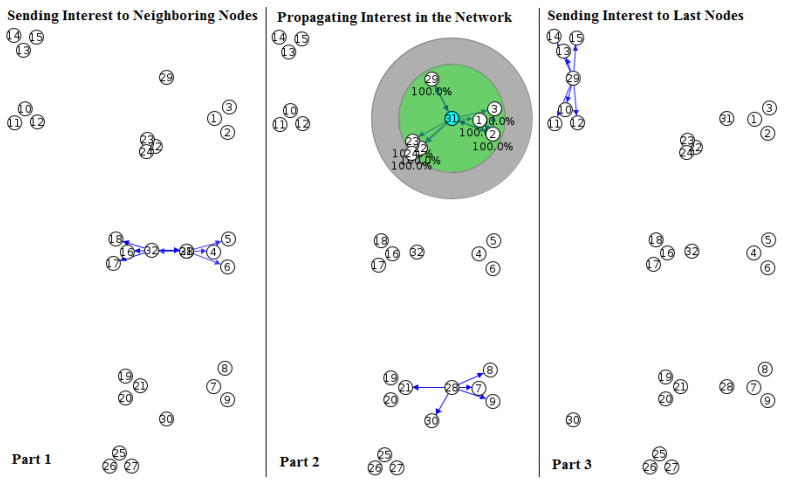

Figure 8. Simulation Scenario 3 (4 Mobile Nodes)

$$
\text { AverageLatency }=\frac{(\text { TotalLatency })}{(\text { TotalPacketsReceived })}
$$

where total latency is the delay between Interest generation by sink node and its reception at the source node and can be calculated using following equation 3 :

$$
\text { TotalLatency }=\sum_{n=1}^{n}(\text { RecvTime }- \text { SentTime })
$$

The aggregation, number of hops and Interest transmissions are computed using the log files generated for the simulations performed.

\section{Results and Discussion}

1) Satisfaction Rate (SR): Because static nodes implement only 'broadcast mode', and broadcast mode is the major reason of 'communication loop' creation. Therefore, due to presence of 'communication loop', low SR is an obvious result and it is evident from Fig. 9 that static nodes exhibit low Interest SR.

As we stated above, we minimize 'communication loop' in scenarios which have mobile nodes by implementing 'unicast mode' in source nodes that reside in departments (i.e., source nodes send data to the requesting mobile node only instead of sending to all neighboring nodes). In this way, the percentage of the average number of Interests satisfied, increased. This results in higher SR. For simulation, a number of Interests are generated and their satisfaction rate is over served by 


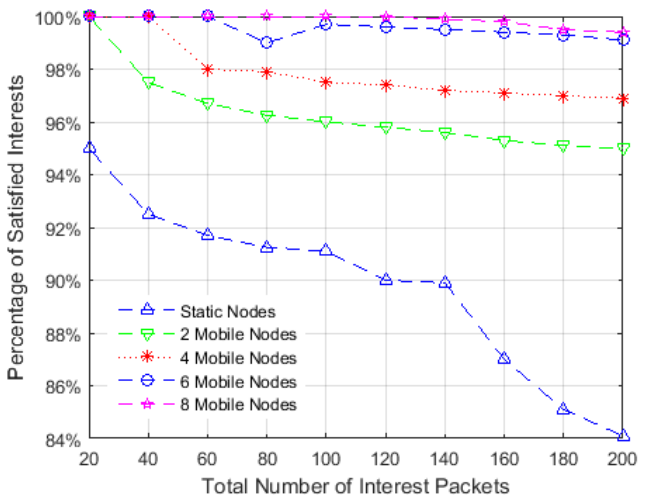

Figure 9. Percentage of satisfied Interests.

receiving the Data requested. Mobile nodes shows better results because more average number of Interests are satisfied before forwarding it to next mobile nodes and it is satisfied before its expiry in the given time period.

2) Average Latency: Average amount of delay in receiving the Interests is higher in mobile nodes scenarios. Reason of this delay is the time which mobile nodes take while traveling from sink node to destination node. Although static nodes show less delay but they have more number of intermediate nodes between the sink and source nodes. Fig. 10 shows average latency computed for different number of Interests in considered scenarios. The average delay in the scenarios with more than two nodes is not much higher because the sink node has some nodes in its range and some on very short distance. With the help of log generated for simulations, it is observed that more Interests are satisfied before forwarding the Interest towards next mobile nodes which result less average delay in comparison to two mobile nodes which move back after completing the path.

3) Transmission of Interest Packets: The average number of transmissions are higher for static nodes because of the greater number of intermediate nodes and broadcasting mode. Every node, which receives the Interest packet, forwards the Interest to neighboring nodes if it does not have the required Data. The mobile nodes work as carrier nodes to take the Interest

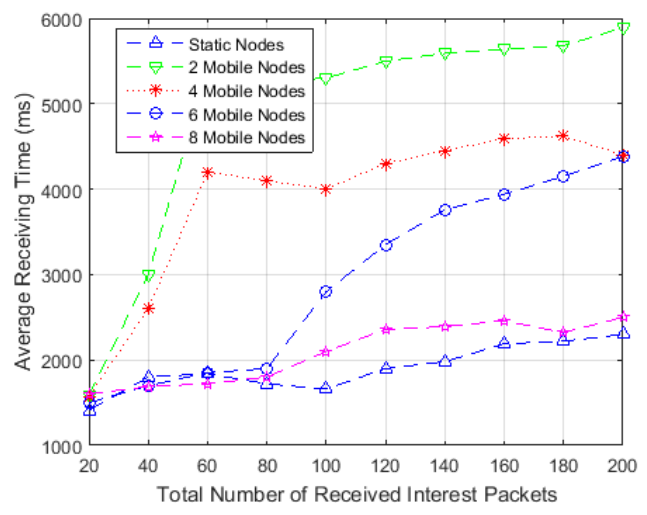

Figure 10. Average delay of receiving Interest.

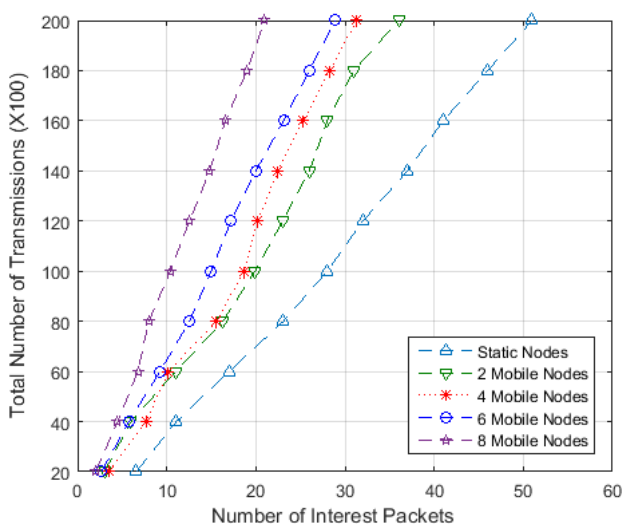

Figure 11. Total number of transmissions (one sink node).

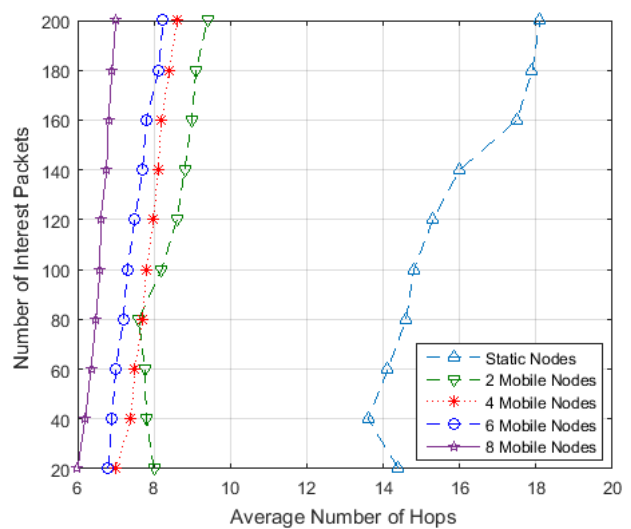

Figure 12. Average number of hops.

packets to nodes at a distance, minimizing the number of hops. They require less number of transmissions because only the mobile nodes works as intermediate nodes. Fig. 11 shows the comparison of transmission of Interest packets to reach the source node.

4) Number of Hops: Fig. 12 highlights and compares the performance of the proposed scheme in terms of number of Interests across different hops. Different number of mobile nodes are presented for detailed investigation. In the case of static nodes, transmission of Interest and Data packets is done with the help of intermediate static nodes only. The sink node broadcasts the Interest to its neighboring nodes. If the Interest is not satisfied, then it is propagated to next nodes until it is satisfied and Data packet is received. For this reason, it has a greater number of hops in comparison to those where mobile nodes transmit Interest and Data packets between the sink and last source node.

5) Interest Aggregation: The aggregation of Interests in the intermediate nodes minimizes the size of routing table (PIT and FIB) and number of entries in it. Fig. 13 shows the percentage of Interests aggregated in considered scenarios. It can be seen that flat naming shows very low aggregation as compared to hierarchical naming. Since, the proposed hybrid-naming scheme has both flat and hierarchical naming components, therefore it has higher Interest aggregation 


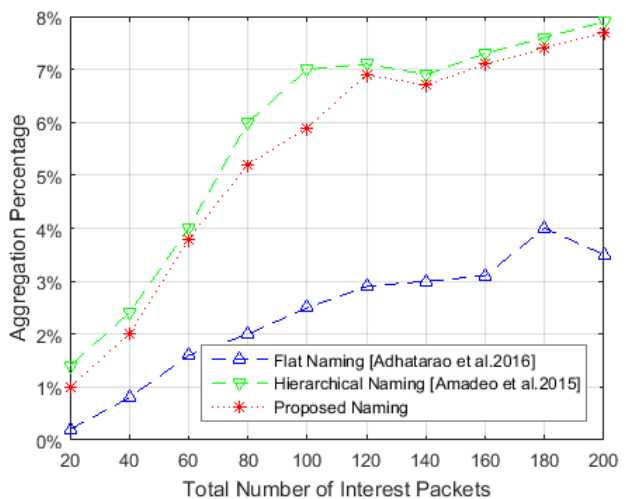

Figure 13. Percentage of Interest aggregation.

in comparison to only the flat naming scheme but less as compared to hierarchical naming. The Interests with similar prefix require only one entry in the routing table and it is differentiated by the flat part, which is unique to every component. The results are obtained by observing that how many Interests are generated and stored in the routing table on average.

\section{CONCLUSION}

This paper presented and simulated a CCN model for IoT based SC application, in which a hybrid naming scheme is proposed where contents are named through the use of both (1) hierarchical and (2) flat naming schemes. The hierarchical component takes the domain name, location, task and device name in URL style. By using 'task', push support is added in the native $\mathrm{CCN}$ protocol. The flat component is used to provide integrity and it is computed through the FNV-1a hash of the device name and Data. The communication loop problem associated with CCN protocol is eliminated by implementing 'unicast' protocol on the source nodes. Mobile IoT nodes are used for delivery of Interest and Data packets to nodes that are not in the range of sink node. The proposed naming scheme is evaluated for IoT-SC having both static and mobile nodes and results revealed the significant gain in terms of success rate and number of transmissions of Interest packets, latency, number of hops and interest aggregation. Furthermore, both attributes and properties of contents and nodes can be incorporated to strengthen the scope of the scheme [23].

\section{ACKNOWLEDGMENT}

This research is supported by the Computer Engineering Department (CPED) of the University of Engineering and Technology (UET), Taxila, Pakistan under a Full-time research scholarship, and in close collaboration with University of West London, UK. The authors would also like to thank the editor and reviewers for their helpful comments and suggestions.

\section{REFERENCES}

[1] D. Evans, "The internet of things how the next evolution of the internet is changing everything (april 2011)," White Paper by Cisco Internet Business Solutions Group (IBSG), 2012.
[2] G. N. Cristina, G. V. Gheorghita, and U. Ioan, "Gradual development of an iot architecture for real-world things," in Modelling Symposium (EMS), 2015 IEEE European. IEEE, 2015, pp. 344-349.

[3] S. JOSE. (2017) Cisco visual networking index predicts near-tripling of ip traffic by 2020. [Online]. Available: https://newsroom.cisco.com/press-release-content?type=pressrelease \&articleId $=1771211$

[4] W. Shang, Y. Yu, R. Droms, and L. Zhang, "Challenges in iot networking via tcp/ip architecture," NDN Project, Tech. Rep. NDN-0038, Tech. Rep., 2016.

[5] G. Xylomenos, C. N. Ververidis, V. A. Siris, N. Fotiou, C. Tsilopoulos, X. Vasilakos, K. V. Katsaros, and G. C. Polyzos, "A survey of information-centric networking research," IEEE Communications Surveys \& Tutorials, vol. 16, no. 2, pp. 1024-1049, 2014.

[6] The ccnx project. [Online]. Available: http://blogs.parc.com/ccnx/

[7] S. H. Ahmed, S. H. Bouk, and D. Kim, Content-centric networks: an overview, applications and research challenges. Springer, 2016.

[8] M. Amadeo, C. Campolo, J. Quevedo, D. Corujo, A. Molinaro, A. Iera, R. L. Aguiar, and A. V. Vasilakos, "Information-centric networking for the internet of things: challenges and opportunities," IEEE Network, vol. 30, no. 2, pp. 92-100, 2016.

[9] Y. Zhang, D. Raychadhuri, L. Grieco, E. Baccelli, J. Burke, and G. Wang, "Icn based architecture for iot - requirements and challenges draft-zhang-iot-icn-challenges-02," ICN Research Group, Internet-Draft, 2016. [Online]. Available: https://tools.ietf.org/html/draft-zhang-iot-icnchallenges-02\#page-12

[10] M. Amadeo, C. Campolo, A. Iera, and A. Molinaro, "Information centric networking in iot scenarios: The case of a smart home," in Communications (ICC), 2015 IEEE International Conference on. IEEE, 2015, pp. 648-653.

[11] S. H. Bouk, S. H. Ahmed, and D. Kim, "Hierarchical and hash based naming with compact trie name management scheme for vehicular content centric networks," Computer Communications, vol. 71, pp. 7383, 2015.

[12] W. Shang, A. Bannis, T. Liang, Z. Wang, Y. Yu, A. Afanasyev, J. Thompson, J. Burke, B. Zhang, and L. Zhang, "Named data networking of things," in Internet-of-Things Design and Implementation (IoTDI), 2016 IEEE First International Conference on. IEEE, 2016, pp. 117-128.

[13] W. Shang, Q. Ding, A. Marianantoni, J. Burke, and L. Zhang, "Securing building management systems using named data networking," IEEE Network, vol. 28, no. 3, pp. 50-56, May 2014.

[14] S. H. Bouk, S. H. Ahmed, and D. Kim, "Ndn goes deep: foreseeing the underwater named data networks," in Proceedings of the Symposium on Applied Computing. ACM, 2017, pp. 642-646.

[15] M. A. M. Hail, M. Amadeo, A. Molinaro, and S. Fischer, "On the performance of caching and forwarding in information-centric networking for the iot," in International Conference on Wired/Wireless Internet Communication. Springer, 2015, pp. 313-326.

[16] E. Baccelli, C. Mehlis, O. Hahm, T. C. Schmidt, and M. Whlisch, "Information centric networking in the iot: Experiments with ndn in the wild," in Proceedings of the 1st international conference on Informationcentric networking. ACM, 2014, pp. 77-86.

[17] S. Li, Y. Zhang, D. Raychaudhuri, and R. Ravindran, "A comparative study of mobilityfirst and ndn based icn-iot architectures," in Heterogeneous Networking for Quality, Reliability, Security and Robustness (QShine), 2014 10th International Conference on. IEEE, 2014, pp. $158-163$.

[18] M. Meisel, V. Pappas, and L. Zhang, "Ad hoc networking via named data," in Proceedings of the fifth ACM international workshop on Mobility in the evolving internet architecture. ACM, 2010, pp. 3-8.

[19] G. Fowler, L. C. Noll, K.-P. Vo, and D. Eastlake, "The fnv noncryptographic hash algorithm," Ietf-draft, 2011.

[20] (2016) Fowler noll vo hash function. [Online]. Available: http://will.thimbleby.net/algorithms/doku.php?id=fowler-noll-vohash-function

[21] B. Saadallah, A. Lahmadi, and O. Festor, "Cenx for contiki: implementation details," Ph.D. dissertation, INRIA, 2012.

[22] H. Lamaazi, N. Benamar, M. I. Imaduddin, and A. J. Jara, "Performance assessment of the routing protocol for low power and lossy networks," in Wireless Networks and Mobile Communications (WINCOM), 2015 International Conference on. IEEE, 2015, pp. 1-8.

[23] S. Arshad, M. A. Azam, S. H. Ahmed, and J. Loo, "Towards information-centric networking (icn) naming for internet of things (iot): The case of smart campus," in Proceedings of the International Conference on Future Networks and Distributed Systems, ser. ICFNDS '17. New York, NY, USA: ACM, 2017, pp. 41:1-41:6. [Online]. Available: http://doi.acm.org/10.1145/3102304.3102345 